American Journal of Applied Sciences 4 (3): 155-159, 2007

ISSN 1546-9239

(C) 2007 Science Publications

\title{
Designing of PID Controller for Discrete Time Linear System Using Balanced Approach Reduced Order Model
}

\author{
${ }^{1}$ C.S.Ravichandran, ${ }^{2}$ S. Subha Rani and ${ }^{3}$ T. Manikandan \\ ${ }^{1}$ Sri Ramakrishna Engineering College, Coimbatore - 641 022, India \\ ${ }^{2}$ P.S.G. College of Technology, Coimbatore - 641 004, India \\ ${ }^{3}$ Kongu Engineering College, Perundurai, Erode- 638 052, India
}

\begin{abstract}
Modeling physical systems usually results in complex high-order dynamic models. It is often desirable to approximate these models by simpler models with reduced order. This study deals with the design of discrete time linear system using a balanced approach reduced order model. The reduced order model retains the desired state variable which contains a significant contribution. A PID controller is designed for the reduced second order model to meet the desired performance specifications by using pole-zero cancellation method. The stabilization of linear discrete time system is achieved by selection of parameters of the PID controller. A numerical example is given to illustrate the design method.
\end{abstract}

Key words: Balanced approach, system order reduction, dominant states, PID controller, discrete time linear system

\section{INTRODUCTION}

Mathematical models of control systems, derived from theoretical considerations, are practically complex and of high order. Such systems are difficult to be analyzed and also the design of controller becomes too difficult. Therefore, efforts are made to have a simplified low-order model which retains the dominant characteristics of the original system. During the last four decades, a significant attention has been paid to construct the reduced order model by retaining only a few modes of linear difference system ${ }^{[1-4]}$. The appropriate definition and determination as to which state variables significantly participate in the selected modes become very important. The significant state variables for an eigen values are those that correspond to large entries in the corresponding eigen vector. But the entries in the eigen vector are dependent on the dimensions of the state variables which are, in general, incommensurable. This study describes the reduced order model for a given discrete time linear system keeping only an essential state of the given plant ${ }^{[5-7]}$. An essential state of a linear system corresponds to the slow modes of the dynamical system. The process of stabilization or improving the performance of the system is achieved by attaching a controller or compensator to the system, either in cascade or in feedback. This study proposes a simple procedure for the design of controllers for a given discrete time systems using pole-zero cancellation method ${ }^{[10,11]}$. To cancel the undesirable poles and zeros of the controller and new open loop poles and zeros are added at more advantageous locations to satisfy the design specifications. The parameters of the controller are tuned to obtain a response with desired performance specifications. The tuned controller is attached with the original higher order system and the closed loop response is observed for stabilization process. The performance of the system with this tuned controller is better than the other tuning procedure.

Balanced approach reduced order model: Consider a linear discrete time system described by the following equation

$$
\left.\begin{array}{l}
x(k+1)=A x(k)+B u(k) \\
y(k)=C x(k)
\end{array}\right\}
$$

The principal component approaches ${ }^{[8,9]}$ are elegant from the point of view of minimal realization; model reduction through subsystem elimination is not a well understood operation.

Let the following balanced transformation $x(k)=T x_{b}(k)$ be applied to the system (1) where $x_{b}(k)$ is an $\mathrm{n}$-dimensional balanced state vector. The internally balanced system is obtained as:

Corresponding Author: $\quad$ C. S. Ravichandran, Sri Ramakrishna Engineering College, Coimbatore - 641 022, India 
$x(k+1)=A^{*} x_{b}(k)+B^{*} u(k)$

$y(k)=C^{*} x_{b}(k)$

Where the system matrices are defined by $A^{*}=T^{-1} A T, B^{*}=T^{-1} B$ and $C^{*}=C T$. Consider the similarity transformations

$$
x(k)=M z(k)
$$

Using normalization transformations (4) on the standard state space equations (2), (3) obtains,

$z(k+1)=\Lambda z(k)+\Gamma u(k)$

$y(k)=F z(k)$

From (5) and (6),

$\left[\begin{array}{l}z_{1}(k+1) \\ z_{2}(k+1)\end{array}\right]=\left[\begin{array}{cc}\Lambda_{1} & 0 \\ 0 & \Lambda_{2}\end{array}\right]\left[\begin{array}{l}z_{1}(k) \\ z_{2}(k)\end{array}\right]+\left[\begin{array}{l}\Gamma_{1} \\ \Gamma_{2}\end{array}\right] u(k)$

$y(k)=\left[\begin{array}{ll}F_{1} & F_{2}\end{array}\right]\left[\begin{array}{l}z_{1}(k) \\ z_{2}(k)\end{array}\right]$

As $k \rightarrow \infty, z_{2}(k+1) \approx z_{2}(k)$, i.e. $z_{2}$ takes a

constant value in the steady state. The matrix $\mathrm{I}-\Lambda_{2}$ is invertible. From (7), for large values of $k$,

$z_{2}(k) \approx \Lambda_{2} z_{2}(k)+\Gamma_{2} u(k)$

i.e, $z_{2}(k) \approx\left(I-\Lambda_{2}\right)^{-1} \Gamma_{2} u(k)$

Using (7), (8) and (9), the reduced-order model in the $z$-coordinates as

$z_{1}(k+1)=\Lambda_{1} z_{1}(k)+\Gamma_{1} u(k)$

$y(k)=F_{1} z_{1}(k)+F_{2}\left(I-\Lambda_{2}\right)^{-1} \Gamma_{2} u(k)$

By the linear change of coordinates (4),

$$
\begin{aligned}
{\left[\begin{array}{c}
z_{1}(k) \\
z_{2}(k)
\end{array}\right] } & =M^{-1}\left[\begin{array}{l}
x_{1}(k) \\
x_{2}(k)
\end{array}\right]=Q\left[\begin{array}{l}
x_{1}(k) \\
x_{2}(k)
\end{array}\right] \\
& =\left[\begin{array}{ll}
Q_{11} & Q_{12} \\
Q_{21} & Q_{22}
\end{array}\right]\left[\begin{array}{l}
x_{1}(k) \\
x_{2}(k)
\end{array}\right]
\end{aligned}
$$

Using (9) and (12),

$$
\begin{aligned}
z_{2}(k) & =Q_{21} x_{1}(k)+Q_{22} x_{2}(k) \\
& \approx\left(I-\Lambda_{2}\right)^{-1} \Gamma_{2} u(k)
\end{aligned}
$$

The matrix $\mathrm{Q}_{22}$ is invertible. The equation (13) may be simplified as:

$$
\begin{aligned}
& x_{2}(k) \approx-Q_{22}^{-1} Q_{21} x_{1}(k)+ \\
& Q_{22}^{-1}\left(I-\Lambda_{2}\right)^{-1} \Gamma_{2} u(k)
\end{aligned}
$$

Using (14), the reduced-order model of the given linear plant as:

$$
\left.\begin{array}{rl}
x_{1}(k+1) & =A_{e} x_{1}(k)+B_{e} u(k) \\
y(k) & =C_{e} x_{1}(k)+D_{e} u(k)
\end{array}\right\}
$$

Where the matrices $A_{e}, B_{e}, C_{e}$ and $D_{e}$ are defined by

$$
\begin{aligned}
& A_{e}=A^{*}{ }_{11}-A^{*}{ }_{12} Q_{22}^{-1} Q_{21} \\
& B_{e}=B^{*}{ }_{1}+A^{*}{ }_{12} Q_{22}^{-1}\left(I-\Lambda_{2}\right)^{-1} \Gamma_{2} \\
& C_{e}=C^{*}{ }_{1}-C^{*}{ }_{2} Q_{22}^{-1} Q_{21} \\
& D_{e}=C^{*}{ }_{2} Q_{22}^{-1}\left(I-\Lambda_{2}\right)^{-1} \Gamma_{2}
\end{aligned}
$$

Equation (15) represents a reduced order representation of the original system.

\section{PID controller design using}

Reduced order model: The design of PID controller for a stable higher system involves a lot of computation. To minimize the difficulty, the higher order system can be reduced to a suitable lower order model using the proposed scheme. The controller is attached with the reduced model and closed loop response is observed. The parameters of the controller are tuned to obtain the response for meeting the specifications. The tuned parameters are introduced into the higher order system for stabilization purposes. In typical control systems, the method of output response stabilization is obtained by the selection of Proportional - Integral - Derivative (PID) controller.

The transfer function of PID controller for discrete system is written as:

$$
\mathrm{G}_{\mathrm{c}}(\mathrm{z})=\mathrm{K}_{\mathrm{p}}+\mathrm{K}_{\mathrm{i}} \cdot \frac{\mathrm{T} . \mathrm{z}}{\mathrm{z}-1}+\mathrm{K}_{\mathrm{d}} \cdot \frac{\mathrm{z}-1}{\mathrm{~T} \cdot \mathrm{z}}
$$

Where $\mathrm{K}_{\mathrm{p}}$ is the proportional gain, $\mathrm{K}_{\mathrm{i}}$ is the integral gain, $\mathrm{K}_{\mathrm{d}}$ is the derivative gain and $\mathrm{T}$ is the sampling time. The design involves the determination of values of the constants $\mathrm{K}_{\mathrm{p}}, \mathrm{K}_{\mathrm{i}}$ and $\mathrm{K}_{\mathrm{d}}$ meeting the required performance specifications. If the system output response does not satisfy the specifications, a PID controller is added to the forward path. In this regard, a PID controller is designed for the reduced model. The initial values of the parameters of PID controllers are obtained by applying pole-zero cancellation method ${ }^{[10,}$ ${ }^{11]}$. The PID controller is attached with the reduced order model and the closed loop output response is observed. If the system does not meet the required specifications, the parameters are tuned to obtain optimum values.

The overall system transfer function as shown in Fig. 1 can be written as: 


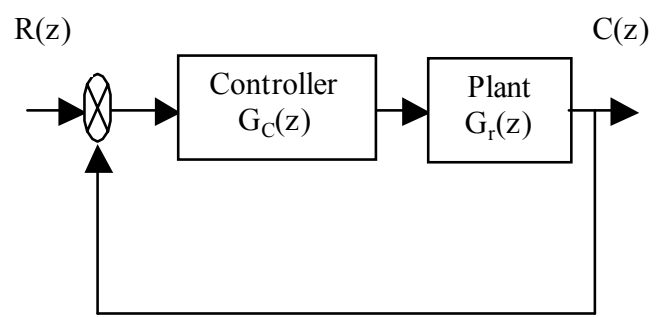

Fig. 1: Block diagram of a compensated system

$$
T F_{C}(z)=\frac{G_{C}(z) \cdot G_{r}(z)}{1+G_{C}(z) \cdot G_{r}(z)}
$$

Where $\mathrm{G}_{\mathrm{c}}(\mathrm{z})$ is the transfer function of the controller, $G_{r}(z)$ is the transfer function of the reduced order plant.

Numerical example: To illustrate the proposed method, consider the $8^{\text {th }}$ order system transfer function ${ }^{[12]}$ given as

$$
G(z)=\frac{1.682 z^{7}+1.116 z^{6}-0.21 z^{5}+0.152 z^{4}-0.516 z^{3}-0.262 z^{2}+0.044 z-0.018}{8 z^{8}-5.046 z^{7}-3.348 z^{6}+0.63 z^{5}-0.456 z^{4}+1.548 z^{3}+0.786 z^{2}-0.132 z+0.018}
$$

The balanced discrete time state space model of given transfer function as:

$$
\begin{aligned}
A^{*} & =\left[\begin{array}{ll}
A_{11} & A_{12} \\
A_{21} & A_{22}
\end{array}\right] ; B^{*}=\left[\begin{array}{l}
B_{1} \\
B_{2}
\end{array}\right] ; C^{*}=\left[\begin{array}{lll}
C_{1} & C_{2}
\end{array}\right] ; D=0 \\
A_{11} & =\left[\begin{array}{llll}
0.8598 & -0.2514 & 0.0535 & -0.0312 \\
0.2514 & 0.8990 & 0.0863 & -0.0414 \\
-0.0535 & 0.0863 & -0.7538 & -0.2688 \\
-0.0312 & 0.0414 & 0.2688 & -0.2938
\end{array}\right] \\
\mathrm{A}_{12} & =\left[\begin{array}{llll}
-0.0261 & 0.0020 & -0.0016 & 0.0001 \\
-0.0363 & 0.0020 & -0.0016 & 0.0001 \\
-0.1644 & 0.0343 & -0.0260 & 0.0012 \\
0.7035 & 0.0811 & -0.0589 & 0.0013
\end{array}\right] \\
\mathrm{A}_{21} & =\left[\begin{array}{llll}
0.0261 & -0.0363 & -0.1644 & -0.7035 \\
0.0020 & -0.0020 & -0.0343 & 0.0811 \\
0.0016 & -0.0016 & -0.0260 & 0.0589 \\
0.0001 & -0.0001 & -0.0012 & 0.0013
\end{array}\right] \\
\mathrm{A}_{22} & =\left[\begin{array}{llll}
0.2789 & -0.1104 & 0.0814 & -0.0025 \\
0.1104 & -0.5437 & -0.4641 & 0.1462 \\
0.0814 & 0.4641 & 0.4963 & 0.2148 \\
0.0025 & 0.1462 & -0.2148 & -0.3119
\end{array}\right]
\end{aligned}
$$

$\mathrm{B}_{1}^{T}=\left[\begin{array}{llll}0.5257 & -0.2200 & 0.1386 & 0.0738\end{array}\right]$

$\mathrm{B}_{2}^{T}=\left[\begin{array}{llll}-0.0632 & -0.0042 & -0.0034 & -0.0003\end{array}\right]$

$\mathrm{C}_{1}=\left[\begin{array}{llll}0.5257 & 0.2200 & -0.1386 & 0.0738\end{array}\right]$

$\mathrm{C}_{2}=\left[\begin{array}{llll}0.0632 & -0.0042 & 0.0034 & -0.0003\end{array}\right]$

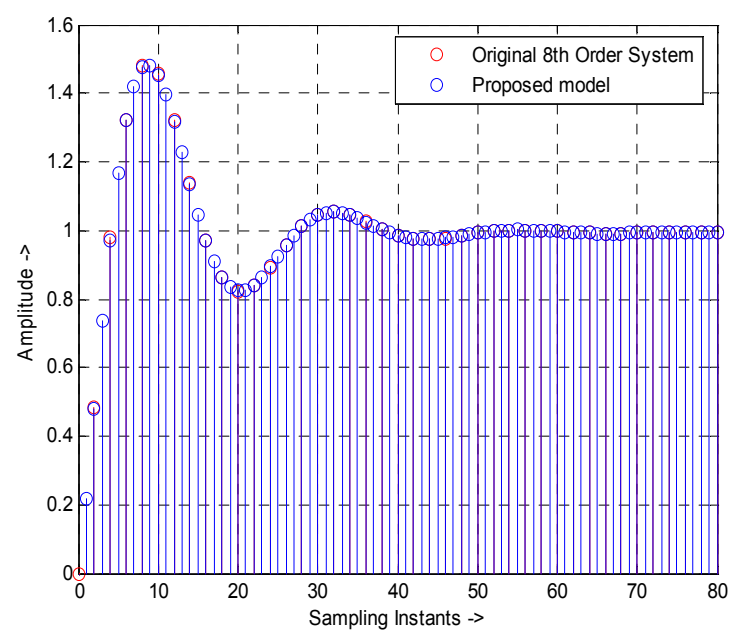

Fig. 2: Step Response of original and reduced order system

The eigen values of the system matrix are

$\lambda_{1,2}=0.8797 \pm 0.2446 \mathrm{i}$

$\lambda_{3,4}=-0.0541 \pm 0.6557 \mathrm{i}$

$\lambda_{5,6}=-0.5876 \pm 0.0955 \mathrm{i}$

$\lambda_{7,8}=0.0773 \pm 0.1078 \mathrm{i}$

From the eigen value contribution measure that the complex conjugate of eigen value $\lambda_{1,2}=0.8797 \pm 0.2446 \mathrm{i}$ is the most dominant of the system. Using (15), the reduced order model is derived for a given discrete time linear system keeping only the dominant state of the given plant as

$$
\begin{aligned}
& \mathrm{A}_{\mathrm{e}}=\left[\begin{array}{cc}
0.8548 & -0.2479 \\
0.2439 & 0.9045
\end{array}\right] ; \mathrm{B}_{\mathrm{e}}=\left[\begin{array}{c}
0.5375 \\
-0.2022
\end{array}\right] ; \\
& \mathrm{C}_{\mathrm{e}}=\left[\begin{array}{ll}
0.5382 & 0.2110
\end{array}\right] ; \mathrm{D}_{\mathrm{e}}=-0.0296
\end{aligned}
$$

Most computational amount is reduced for this reduction method and also for implementing in the applications of large scale interconnected systems. 
By applying the proposed scheme, the reduced order model is obtained and given as:

$$
G_{r}(z)=\frac{-0.02962 \mathrm{z}^{\wedge} 2+0.2988 \mathrm{z}-0.1953}{\mathrm{z}^{\wedge} 2-1.7593 \mathrm{z}+0.8336}
$$

Applying pole-zero cancellation method to the reduced model, the initial values of $\mathrm{K}_{\mathrm{p}}, \mathrm{K}_{\mathrm{i}}$ and $\mathrm{K}_{\mathrm{d}}$ are obtained as:

$$
\begin{aligned}
\mathrm{K}_{d} & =0.8336 \\
-2 \mathrm{~K}_{d}-\mathrm{K}_{\mathrm{p}} & =-1.7593 \\
\mathrm{~K}_{\mathrm{p}}+\mathrm{K}_{\mathrm{d}}+\mathrm{K}_{\mathrm{i}} & =1 \\
\text { i.e. } \mathrm{K}_{\mathrm{p}}=0.0921, \mathrm{~K}_{\mathrm{i}} & =0.0743, \mathrm{~K}_{\mathrm{d}}=0.8336
\end{aligned}
$$

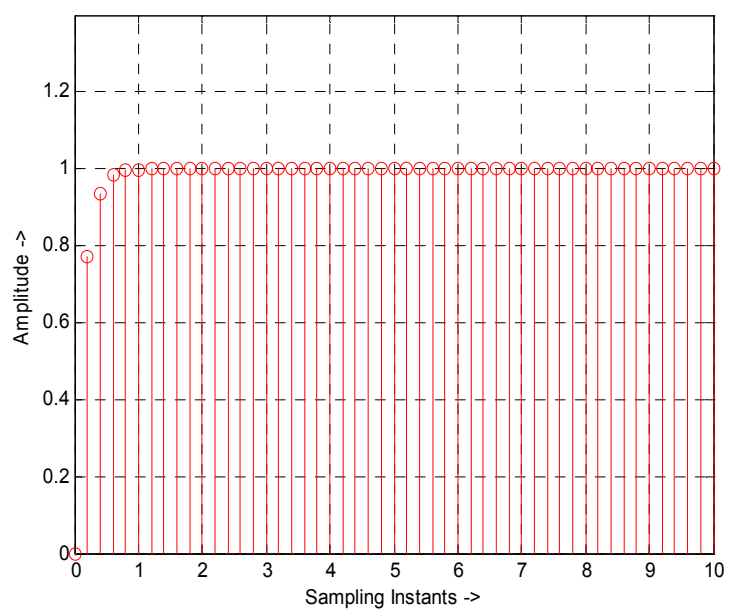

Fig. 3: Step response of original system with PID controller

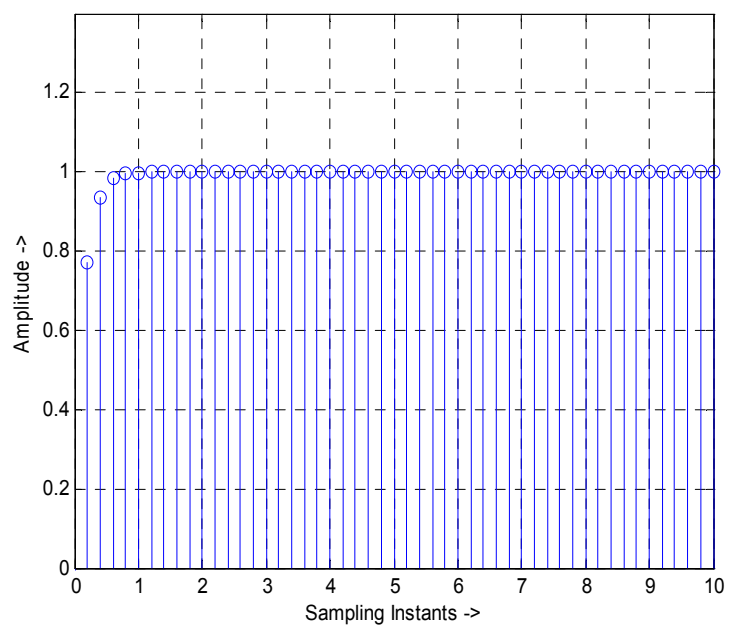

Fig. 4: Step response of reduced order model with PID controller
Using the simulation procedure, the initial parameters are tuned with $T=0.01 \mathrm{sec}$ to get the step response of the compensated system to meet the required specifications. The designed PID controller $\mathrm{G}_{\mathrm{c}}(\mathrm{z})$ is attached with the original higher order system. Figure 3 and 4 show the step response of the reduced and higher order system with PID controller.

\section{CONCLUSION}

This study proposes the balanced approach reduced order memory less state space linear discrete time system. The essential part of the state variables of the system is used for the reduction of discrete time systems to obtain stable low order models. The proposed model reduction scheme is suitably extended to the design of PID Controllers for Discrete time system. This approach minimizes the complexity involved in direct design of PID Controller. The approximate values for PID Controller are calculated from the reduced order model and suitably tuned to meet the required performance specifications. The tuned values of these controller parameters are attached with the original system and its closed loop response for a unit step input is found to be in good accord with the response of reduced order model.

\section{REFERENCES}

1. Fortman, T.E. and D. Williamson, 1972. Design of low-order observers for linear feedback control laws. IEEE Trans. on Automatic Control, AC-17: 301-308.

2. Davison, E.J., 1966. A method for simplifying linear dynamic systems. IEEE Trans. on Automatic Control, AC-11: 93-101.

3. Lastman, G.J., N.K. Sinha and P. Rozsa, 1984. On the selection of states to be retained in a reducedorder model. IEE Proc. Control Theory, 131: 1524.

4. Aldeen, M. and H. Trinh, 1994. Observing a subset of states of linear systems. IEE Proc.-Control Theory, 141: 137-144.

5. Ravichandran, S. and S. Subha Rani, 2003. Observers for the dominant states of linear difference system. Intl. Conf. on Robotics, Vision, Informatics and Signal Processing, Jan. 22-24, pp: 467-471, Penang, Malaysia.

6. Ravichandran, S. and S. Subha Rani, 2004. Reduced order controllers for stabilization of large scale linear difference systems. Intl. Conf. on Systematics, Cybernetics and Informatics, Pentagram Research Centre Pvt. Ltd, Feb. 12-15, pp: 246-250, Hyderabad. 
7. Ravichandran, C.S., Subha Rani, S., Sundarapandian, V., 2006. Simple Method for Design of Reduced Order Output Feedback Controller for Discrete Time Linear System, Gest International Transaction on Communication and Signal Processing, Vol.9, No.1, pp.63-74.

8. Moore, B.C., 1981. Principal component analysis in linear system: Controllability, observability and model reduction. IEEE Trans. Automatic Control, AC-26: 17-32.

9. Laub, A.J., 1980. Computation of balancing transformations. Proc. of the Joint Automatic Control Conf., F48-E
10. Manigandan, T., N. Devarajan and S.N. Sivanandam, 2005. Design of PID controller using reduced order model. Academic Open Internet J., Vol. 15, Part 1.

11. Kuo, B.C., 1982. Automatic Control Systems. Prentice Hall of India, New Delhi, India.

12. Prasad R., 1993. Order reduction of discrete time using stability equation method and weighted time moments. Journal of I.E (India), Vol.74, pp. 94 -99. 